\title{
The Role of Alveolar Macrophages in Pneumocystis carinii Degradation and Clearance from the Lung
}

\author{
Andrew H. Limper, ${ }^{\star} \neq$ Jacques S. Hoyte, ${ }^{\star}$ and Joseph E. Standing ${ }^{\star}$ \\ *Thoracic Diseases Research Unit, Division of Pulmonary, Critical Care and Internal Medicine, Department of Medicine, and \\ ${ }^{\ddagger}$ Department of Biochemistry and Molecular Biology, Mayo Clinic, Rochester, Minnesota 55905
}

\begin{abstract}
Although studies indicate that alveolar macrophages participate in host defense against Pneumocystis carinii, their role in organism degradation and clearance from the lung has not yet been established. We, therefore, quantified the uptake and degradation of ${ }^{35} \mathrm{~S}$-labeled $P$. carinii by cultured macrophages, demonstrating significant degradation of $P$. carinii over $6 \mathrm{~h}$. We further evaluated the role of macrophages in elimination of $P$. carinii from the living host. Rats received either intratracheal PBS, liposomal PBS (L-PBS), or liposomal dichloromethylene diphosphonate ( $\left.\mathrm{L}-\mathrm{Cl}_{2} \mathrm{MDP}\right)$, a preparation which leads to selective depletion of macrophages. Over $72 \mathrm{~h}, \mathrm{~L}-\mathrm{Cl}_{2} \mathrm{MDP}-$ treated animals had loss of $>85 \%$ of their alveolar macrophages. In contrast, L-PBS-treated rats had cellular differentials identical to rats receiving PBS. Macrophage-depleted rats and controls were next inoculated with $P$. carinii and organism clearance was determined after 24 h. $P$. carinii elimination was evaluated with both cyst counts and an ELISA directed against glycoprotein A (gpA), the major antigen of $P$. carinii. Both assays indicated that macrophage-depleted rats had substantial impairment of $P$. carinii clearance compared to L-PBS- or PBS-treated rats. These data provide the first direct evidence that macrophages mediate elimination of $P$. carinii from the living host. (J. Clin. Invest. 1997. 99:2110-2117.) Key words: Pneumocystis carinii • macrophage • liposome • phagocytosis • bisphosphonates
\end{abstract}

\section{Introduction}

Pneumocystis carinii remains a significant cause of life-threatening pneumonia in immunocompromised patients, particularly those with AIDS, hematological or solid malignancies, organ transplantation, or inflammatory conditions treated with immunosuppressive agents (1-8). Host defenses against $P$. carinii are poorly understood, but involve a number of cellular mediators, most notably CD4 lymphocytes whose depletion promotes development of $P$. carinii pneumonia $(9,10)$. Additional

Address correspondence to Andrew H. Limper, Thoracic Diseases Research Unit, 601A Guggenheim Building, Mayo Clinic and Foundation, Rochester, MN 55905. Phone: 507-284-2301; FAX: 507-2844521; E-mail: limper.andrew@mayo.edu

Received for publication 6 November 1996 and accepted in revised form 13 February 1997.

J. Clin. Invest.

(C) The American Society for Clinical Investigation, Inc. 0021-9738/97/05/2110/08 \$2.00

Volume 99, Number 9, May 1997, 2110-2117 studies implicate roles for alveolar macrophages, CD8 lymphocytes, and neutrophils in host defense during $P$. carinii pneumonia (11-16).

Accumulating evidence indicates that alveolar macrophages provide a number of key functions during $P$. carinii infection $(12,13,17-23)$. Ultrastructural and in vitro experiments demonstrate that macrophages bind and internalize $P$. carinii $(12,13,17,20,24)$. Macrophage mannose receptors mediate uptake of the organisms through interaction with glycoprotein A (gpA) ${ }^{1}$ a $120-\mathrm{kD}$ antigenic complex present on the surface of $P$. carinii $(17,20,25-32)$. Additional in vitro studies demonstrate that interaction of $P$. carinii with alveolar macrophages causes release of proinflammatory substances including reactive oxidants, arachidonic acid metabolites, and TNF- $\alpha(18,19$, $21,33,34)$.

The role of alveolar macrophages in $P$. carinii degradation and clearance from the lungs has not yet been established. This study was, therefore, undertaken to quantify the binding, uptake, and degradation of $P$. carinii organisms by macrophages. We further provide evidence that alveolar macrophages mediate degradation and clearance of $P$. carinii organisms from the intact host.

\section{Methods}

Materials. Monoclonal antibody 5E12 recognizing $P$. carinii gpA was provided by Dr. Frank Gigliotti (University of Rochester, Rochester, New York) (35). Concanavalin A conjugated to horseradish peroxidase was obtained from E.Y. Laboratories, Inc. (San Mateo, CA). Ciprofloxacin was from Miles Pharmaceuticals (West Haven, CT). Initial samples of liposomal dichloromethylene diphosphonate were a kind gift from Drs. Yongmoon Han and Jim Cutler (Montana State University, Bozeman, MT) (36). Pamidronate was purchased from Ciba-Geigy Corp. (Suffern, NY).

Preparation of P. carinii. All animal studies were approved by the institutional animal care and utilization committee. P. carinii pneumonia was induced in Harlan Sprague-Dawley rats by immunosuppression with dexamethasone and transtracheal injection with $P$. carinii (37-40). Pathogen-free rats were provided with drinking water containing dexamethasone ( $2 \mathrm{mg} /$ liter $)$, tetracycline (500 mg/liter), and nystatin (200,000 U/liter) and fed an $8 \%$ protein diet (Teklad Premier Laboratory Diets, Madison, WI). Each week, the animals received oral ciprofloxacin $(0.45 \mathrm{~g} /$ liter $)$ for two consecutive days to reduce the risk of bacterial infections (41). After $5 \mathrm{~d}$ of immunosuppression, rats were transtracheally inoculated with $P$. carinii $(\sim 500,000$ cysts $)$ prepared by homogenizing infected rat lung. The rats were suppressed for an additional 4-8 wk, killed, and the lungs homogenized in $50 \mathrm{ml}$

1. Abbreviations used in this paper: BAL, bronchoalveolar lavage; $\left[{ }^{14} \mathrm{C}\right]$ UDPG, [glucose- $-{ }^{14} \mathrm{C}$ ] uridine diphosphate glucose; gpA, the glycoprotein A surface antigenic complex of $P$. carinii; $\mathrm{L}_{-} \mathrm{Cl}_{2} \mathrm{MDP}$, liposomal dichloromethylene diphosphonate; L-PAM, liposomal pamidronate disodium; L-PBS, liposomal PBS. 
of HBSS using a laboratory blender. $P$. carinii were purified by differential filtration. The homogenates were exhaustively filtered through $10-\mu \mathrm{M}$ filters which retain lung cells but allow passage of $P$. carinii organisms. The filtrates were collected, centrifuged (1,500 $\mathrm{g}$ for $30 \mathrm{~min})$, and the pellets resuspended in $5 \mathrm{ml}$ of HBSS. Duplicate $10 \mu \mathrm{l}$ aliquots of suspension were spotted onto slides, stained with Diff Quick, and $P$. carinii quantified as described $(40,42)$. If other microorganisms were noted in the smear or on microbiologic culture the material was discarded.

Preparation of gpA and monoclonal antibody to gpA. The major surface antigenic complex of $P$. carinii, gpA, was purified by continuous-flow gel electrophoresis as we previously reported $(20,22)$. In brief, isolated $P$. carinii were solubilized in $125 \mathrm{mM}$ Tris, $4 \%$ SDS, $4 \%$ 2-mercaptoethanol, $0.002 \%$ bromophenol blue, and $20 \%$ glycerol, pH 7.4. This extract was separated on a $10 \%$ polyacrylamide preparative tube gel (PrepCell Apparatus; Biorad, Hercules, CA). The gel was resolved over $48 \mathrm{~h}$ using 35-mA current and eluted with $25 \mathrm{mM}$ Tris Base, $192 \mathrm{mM}$ glycine, and 0.1\% SDS. Fractions were analyzed by SDS-PAGE and silver staining on $4-15 \%$ gradient resolving gels. Fractions containing the complex migrating at $120 \mathrm{kD}$ were pooled, dialyzed, and concentrated. Immunoblotting of the purified gpA complex was performed using monoclonal antibody $5 \mathrm{E} 12$ to verify the identity of the product $(20,35)$. Monoclonal $5 \mathrm{E} 12$ is a mouse anti-P carinii IgM-recognizing gpA from rodents, ferrets, and humans (35).

A second monoclonal antibody, LM-1, recognizing gpA was generated in the following manner. Balb/c mice were immunized with purified gpA and the spleens fused with SP2/OAg14 myeloma cells using previously published protocols $(43,44)$. Fusions were cultured in 96-well plates in the presence of hypoxanthine aminopterin thymidine (HAT) selection medium, and growth-positive wells assessed by ELISA to gpA. Antibody-positive colonies were cloned by limiting dilution, recloned antibody secreting cells expanded in tissue culture, and grown as ascites tumors in pristane-primed Balb/c mice. Ascites fluid was harvested and treated with protease inhibitors including $2 \mathrm{mM}$ PMSF, $10 \mathrm{mM}$ EDTA, and $1 \mathrm{mM} N$-ethyl maleimide. Monoclonal IgG was purified from the ascites by protein A-Sepharose affinity chromatography.

Determination of $P$. carinii binding, uptake, degradation, and viability. The initial binding of radiolabeled $P$. carinii to macrophages was quantified as follows. Alveolar macrophages $\left(1 \times 10^{6}\right)$ were plated overnight in 24-well tissue culture dishes using mixed medium (RPMI/ medium $199,1: 1)$ containing $20 \% \mathrm{FBS}$ at $37^{\circ} \mathrm{C}$. P. carinii were radiolabeled by incubating organisms $\left(400 \times 10^{6}\right)$ for $18 \mathrm{~h}$ in $2 \mathrm{ml} \mathrm{DME}$ with $250 \mu \mathrm{Ci}\left[{ }^{35}\right.$ S $]$ cysteine/methionine (Trans Label, NEN Research Products, Boston, MA). Next, radiolabeled P. carinii were washed with DME and BSA $(1 \mathrm{mg} / \mathrm{ml})$ to remove unincorporated label. ${ }^{35} \mathrm{~S}$ labeled $P$. carinii $\left(4 \times 10^{6} /\right.$ well $)$ were then allowed to attach to macrophages in DME and BSA $(1 \mathrm{mg} / \mathrm{ml})$ at $37^{\circ} \mathrm{C}$ for $0-6 \mathrm{~h}$. Unattached $P$. carinii were removed by washing, and the percentage of organisms associated with macrophages quantified.

To quantify $P$. carinii uptake and degradation by macrophages, $P$. carinii were similarly labeled overnight with $\left[{ }^{35} \mathrm{~S}\right]$ cysteine/methionine $\left(250 \mu \mathrm{Ci},{ }^{35} \mathrm{~S}\right.$-Trans label; NEN $)$ and washed to remove unincorporated label. ${ }^{35} \mathrm{~S}-P$. carinii were allowed to bind to the macrophages over 0-6 h. $P$. carinii uptake was determined as follows. Nonbound $P$. carinii were removed by washing. Bound but nonphagocytized $P$. carinii were removed from the macrophages by digestion with trypsin $(2 \mu \mathrm{g} / \mathrm{ml})$ in EDTA $(0.2 \mathrm{mg} / \mathrm{ml})$ for $15 \mathrm{~min}$ on ice. The macrophages were then separated from the liberated $P$. carinii by differential centrifugation in which the macrophages, but not the released $P$. carinii, were pelleted at $400 \mathrm{~g}$ for $5 \mathrm{~min}$. Phagocytized $P$. carinii were counted in the macrophage pellet. The supernatant was spun again (2,000 $\mathrm{g}$ for $10 \mathrm{~min})$ to bring down nonbound P. carinii. P. carinii degradation was determined by the amount of soluble ${ }^{35} \mathrm{~S}$-label released into the medium. To correct for spontaneous release of ${ }^{35} \mathrm{~S}$-label from $P$. carinii, radiolabeled organisms were treated in an identical fashion without macrophages. Spontaneous release of ${ }^{35} \mathrm{~S}$ was sub- tracted from the amount released in the presence of macrophages to yield a net measurement of $P$. carinii degradation.

Additional experiments were undertaken to determine whether alveolar macrophages suppress $P$. carinii viability in culture. Alveolar macrophages $\left(0.5 \times 10^{6}\right)$ were plated overnight in 48 -well tissue culture dishes in mixed medium with $20 \% \mathrm{FBS}$ at $37^{\circ} \mathrm{C}$. The next day, the macrophages were rinsed with DME containing BSA $(1 \mathrm{mg} / \mathrm{ml})$ and incubated with $P$. carinii organisms $\left(4 \times 10^{6}\right)$ in DME containing BSA for $6 \mathrm{~h}$ at $37^{\circ} \mathrm{C}$. Bound $P$. carinii were released from the macrophages by treatment with trypsin as described above. In addition, internalized $P$. carinii were released by freezing and thawing the specimens twice and by passage through a 23 -gauge needle. Next, $P$. carinii were separated free of the macrophage component by differential centrifugation in which the macrophage component was pelleted at $400 \mathrm{~g}$ for $5 \mathrm{~min}$, and the free $P$. carinii subsequently collected by centrifugation at 2,000 $g$ for 10 minutes. Control $P$. carinii were cultured in the absence of macrophages, and subjected to identical digestion, freeze-thaw, and centrifugation conditions. Subsequently, $P$. carinii viability was determined by the ability of aliquots of $P$. carinii to incorporate [glucose- $\left.{ }^{14} \mathrm{C}\right]$ uridine diphosphate glucose $\left(\left[{ }^{14} \mathrm{C}\right] \mathrm{UDPG}\right.$; NEC 403; NEN) into cell wall $\beta$-glucan (45). Following the differential centrifugation, the $P$. carinii pellets were resuspended in $30 \mu \mathrm{l}$ of $50 \mathrm{mM}$ Hepes, containing $0.05 \%$ sodium deoxycholate, $\mathrm{pH} 7.40$. To each 30- $\mu \mathrm{l}$ aliquot was added $20 \mu \mathrm{l}$ of $1 \mathrm{mM}$ cold UDPG and $1 \mathrm{mM}$ EDTA containing $0.25 \mu \mathrm{Ci}$ of $\left[{ }^{14} \mathrm{C}\right] \mathrm{UDPG}$. The reactions were incubated at $37^{\circ} \mathrm{C}$ for $4 \mathrm{~h}$. Aliquots were spotted and dried onto glass filter paper discs, washed twice with $20 \%$ trichloroacetic acid, twice with dry acetone, and counted. To verify that macrophage components were not significantly contaminating the final preparation nor contributing to measured incorporation of $\left[{ }^{14} \mathrm{C}\right] \mathrm{UDPG}$, macrophages were also cultured in the absence of $P$. carinii and processed in an identical manner.

Generation of liposomal bisphosphonate compounds. Liposomes containing bisphosphonates are selectively phagocytized by alveolar macrophages resulting in depletion of these cells from the lung (46). Liposomes were generated containing either dichloromethylene diphosphonate or pamidronate disodium according to the method of Van Rooijen (47). In brief, multilamellar liposomes were formed from $75 \mathrm{ml}$ of $\mathrm{L}-\alpha$-phosphatidyl choline $(100 \mathrm{mg} / \mathrm{ml})$ and $11 \mathrm{ml}$ of cholesterol $(100$ $\mathrm{mg} / \mathrm{ml}$ ) dissolved in methanol and chloroform (1:1 vol). After removal of the solvent by low-vacuum rotary evaporation, the lipid film was dissolved in chloroform $(10 \mathrm{ml})$. The thin lipid film formed after a second evaporation was dispersed for $15 \mathrm{~min}$ in $10 \mathrm{ml}$ of PBS containing either $1.89 \mathrm{~g}$ of dichloromethylene diphosphonate to form liposomal dichloromethylene diphosphonate $\left(\mathrm{L}-\mathrm{Cl}_{2} \mathrm{MDP}\right)$ liposomes or $120 \mathrm{mg}$ of pamidronate disodium to form liposomal pamidronate disodium (L-PAM) liposomes, respectively. These suspensions were subjected to four freeze-thaw cycles, incubated for $2 \mathrm{~h}\left(25^{\circ} \mathrm{C}\right)$, and sonicated for $3 \mathrm{~min}$. After incubation for an additional $2 \mathrm{~h}$, the liposomes were diluted in PBS $(90 \mathrm{ml})$ and centrifuged at $100,000 \mathrm{~g}$ for $30 \mathrm{~min}$. The liposomes were either used immediately or stored under nitrogen at $4^{\circ} \mathrm{C}$ for up to $2 \mathrm{wk}$. For control liposomes, liposomes were formed in the presence of PBS alone in an identical fashion (liposomal PBS [L-PBS]).

Role of alveolar macrophages in $P$. carinii clearance in vivo. Rats were administered equipotent doses of either $\mathrm{L}-\mathrm{CL}_{2} \mathrm{MDP}(0.2 \mathrm{ml})$ or L-PAM $(0.6 \mathrm{ml})$ or corresponding volumes of PBS or L-PBS by intratracheal installation $(48,49)$. After $72 \mathrm{~h}$, macrophage depletion was assessed by whole-lung lavage $(50 \mathrm{ml})$. The lavage was processed and differential counts performed on at least 200 consecutive cells on cytopreparation smears (50). Initial studies indicated that liposomal bisphosphonate treatment eliminated most alveolar macrophages from the lavage resulting in the formation of morphologically distinct remnant macrophages. Remnant macrophages were defined as those cells which did not possess clear evidence of cytoplasm and a limiting cellular membrane, and characteristically consisted of only a condensed nuclear body.

To evaluate the role of macrophages in $P$. carinii clearance from 
an intact host, rats were depleted of alveolar macrophages by intratracheal instillation of L-CL $\mathrm{C}_{2} \mathrm{MDP}$, L-PBS, or PBS $(0.2 \mathrm{ml})$. After $72 \mathrm{~h}$, the animals were challenged transtracheally with $P$. carinii $\left(1 \times 10^{7}\right)$. After an additional $24 \mathrm{~h}$, rats were killed and whole-lung lavage performed with $50 \mathrm{ml}$ of HBSS. The BAL was centrifuged ( $800 \mathrm{~g}$ for 10 $\mathrm{min}$ ) to pellet inflammatory cells and associated $P$. carinii. Pellets were resuspended in $1 \mathrm{ml}$ of HBSS. Cytopreparation smears were prepared from equivalent quantities of BAL suspension $(150 \mu \mathrm{l})$ and stained with methenamine silver to visualize $P$. carinii systs. Cyst burdens were counted in 40 contiguous oil immersion fields from each slide as previously described (1). The coefficient of variation for this method is $\sim 7.5 \%$ (1).

Determination of total P. carinii gpA by ELISA. As a further measure of $P$. carinii degradation and clearance from the lung, an ELISA was developed which measures total $P$. carinii gpA present in specimens. Animals received intratracheal injection of either PBS, L-PBS, or L-PAM ( $0.6 \mathrm{ml}$ each). After $72 \mathrm{~h}$ of macrophage depletion, the animals were transtracheally inoculated with $P$. carinii $\left(1 \times 10^{7}\right)$. After an additional $24 \mathrm{~h}$, animals underwent BAL $(50 \mathrm{ml})$, and the BAL centrifuged ( $800 \mathrm{~g}$ for $10 \mathrm{~min}$ ) to pellet inflammatory cells and associated $P$. carinii. The pellets were dissolved in $1 \%$ SDS with $15 \mathrm{mM}$ dithiothreitol. Subsequently, samples were treated with iodoacetamide $(30 \mathrm{mM})$, and mixed micelles formed by adding $1 \%$ Triton X-100 (4:1 by vol). The gpA contained in the samples was quantified by capture ELISA. Monoclonal antibody recognizing gpA (monoclonal LM-1; $15 \mu \mathrm{g} / \mathrm{ml}$ in $0.1 \mathrm{M} \mathrm{NaHCO}_{3}$ ) was coated onto plastic plates overnight at $4^{\circ} \mathrm{C}$. The plates were washed and blocked with $1 \%$ TWEEN-20 in TBS for $1 \mathrm{~h}$. After washing, samples were applied to the wells, incubated at room temperature for $2 \mathrm{~h}$, and washed extensively. Next, the wells were treated with concanavalin A conjugated to horseradish peroxidase $(25 \mu \mathrm{g} / \mathrm{ml})$ for $2 \mathrm{~h}$, again washed, and the ELISA developed with $o$-phenylene diamine substrate.

Statistical analysis. Differences between multiple data groups were first assessed by ANOVA. Paired data groups were compared using Student's $t$ tests. Statistical testing was performed using the Statview II statistical package (Abacus Concepts, Inc., Berkeley, CA), with $P \leq 0.05$ defining a statistically significant difference.

\section{Results}

Alveolar macrophages bind, take up, and degrade, $P$. carinii in culture. We first determined the ability of cultured alveolar macrophages to mediate the binding, uptake, and degradation of $P$. carinii (Fig. 1). P c carinii were radiolabeled and macrophage-mediated binding, uptake, and degradation of $P$. carinii determined simultaneously. At all time points beyond $60 \mathrm{~min}$ the cultured alveolar macrophages exhibited significant binding, uptake, and degradation of $P$. carinii $(P<0.05$ each compared to that parameter measured at time zero). Each parameter increased over the $6 \mathrm{~h}$ of study. These data demonstrate that normal alveolar macrophages in culture mediate not only the binding and uptake of $P$. carinii, but also degrade the organisms.

Additional experiments were performed to further determine whether alveolar macrophages suppress $P$. carinii viability. $P$. carinii viability was determined by the ability of $P$. carinii to incorporate $\left[{ }^{14} \mathrm{C}\right] \mathrm{UDPG}$ into cell wall $\beta$-glucan after incubation in the presence or absence of macrophages. Over $6 \mathrm{~h}$ of culture, macrophages substantially reduced the viability of $P$. carinii by $78.1 \pm 15.2 \%$ ( $P=0.001$ compared to control $P$. carinii viability in the absence of macrophages). Macrophages cultured in the absence of $P$. carinii, were lysed and submitted to identical differential centrifugation separation, yielding negligible product and no significant incorporation of $\left[{ }^{14} \mathrm{C}\right] \mathrm{UDPG}$. These experiments demonstrate that macrophages suppress

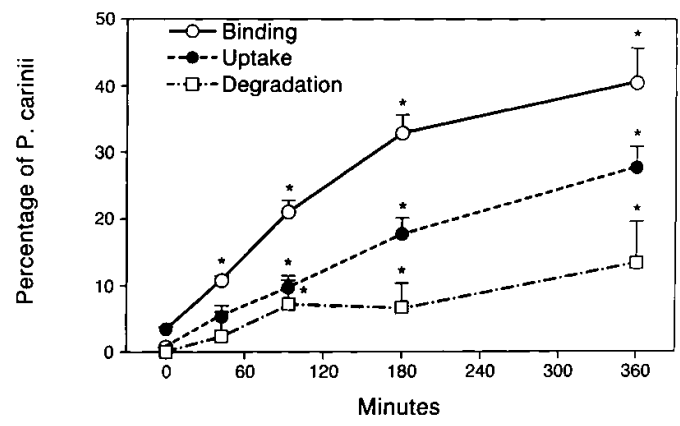

Figure 1. Alveolar macrophages mediate uptake and degradation of $P$. carinii. To determine whether alveolar macrophages mediate uptake and degradation of $P$. carinii, organisms were ${ }^{35} \mathrm{~S}$-labeled and allowed to interact with normal alveolar macrophages in culture. At the times specified, the percentage of $P$. carinii bound (cpm associated with the macrophages after washing), percentage of $P$. carinii phagocytized (cpm not released after trypsin digestion), and the percentage of $P$. carinii degraded (free cpm released into the media) were determined. At all times after $60 \mathrm{~min}, P$. carinii binding, uptake, and degradation were statistically greater than at time zero. $(* P<0.05)$. Shown is mean \pm SEM of four determinations.

$P$. carinii viability and further confirm that alveolar macrophages eliminate $P$. carinii in culture.

Liposomal bisphosphonates deplete alveolar macrophages from the lung. Liposomal administration of bisphosphonates has been used to selectively deplete macrophages and thereby study their function in a variety of experimental settings (36, 46-48). Macrophages phagocytized lecithin-cholesterol liposomes, the bisphosphonate is released, chelating intracellular calcium, and thereby resulting in the death of the cell. To study the role of alveolar macrophages in mediating clearance of $P$. carinii from the lower respiratory tract of rats, liposomes containing dichoromethylene bisphosphonate ( $\left.\mathrm{L}-\mathrm{Cl}_{2} \mathrm{MDP}\right)$ or pamidronate disodium (L-PAM) were administered to normal rats. Administration of both agents effectively eliminated most of the recoverable alveolar macrophages as assessed by whole-lung BAL. Cellular differentials counts obtained from $\mathrm{L}-\mathrm{Cl}_{2} \mathrm{MDP}$-treated animals contained only $12.7 \pm 1.2 \%$ intact alveolar macrophages, compared to $95.3 \pm 0.9 \%$ intact macrophages in PBS-treated animals $(P=0.0001$; Fig. $2, A, C$, and $E$, and Fig. 3). L-PBS animals exhibited cellular differential counts virtually identical to PBS treated animals. Interestingly, depletion of alveolar macrophages with liposomal dichloromethylene bisphosphonate did not induce a significant recruitment of neutrophils $\left(4.0 \pm 2.5 \%\right.$ in $\mathrm{L}_{-} \mathrm{Cl}_{2} \mathrm{MDP}$ treated rats versus $2.3 \pm 1.3 \%$ in PBS controls, $P=0.59$ ).

Treatment of rats with equipotent doses of L-PAM yielded similar degrees of macrophage depletion. L-PAM treated animals had $17.1 \pm 4.5 \%$ intact alveolar macrophages, $72.4 \pm 5.3 \%$ remnant macrophages, $8.1 \pm 1.1 \%$ neutrophils, $1.3 \pm 1.0 \%$ lymphocytes, and $1.0 \pm 0.7 \%$ eosinophils. Taken together, these data indicate that liposomal bisphosphonates selectively eliminate the majority of alveolar macrophages.

These studies indicated that liposomal bisphosphonate treatment eliminates most alveolar macrophages from the lavage and results in the formation of remnant macrophages. Remnant macrophages were defined as those cells that did not possess clear evidence of cytoplasm or a limiting cellular mem- 

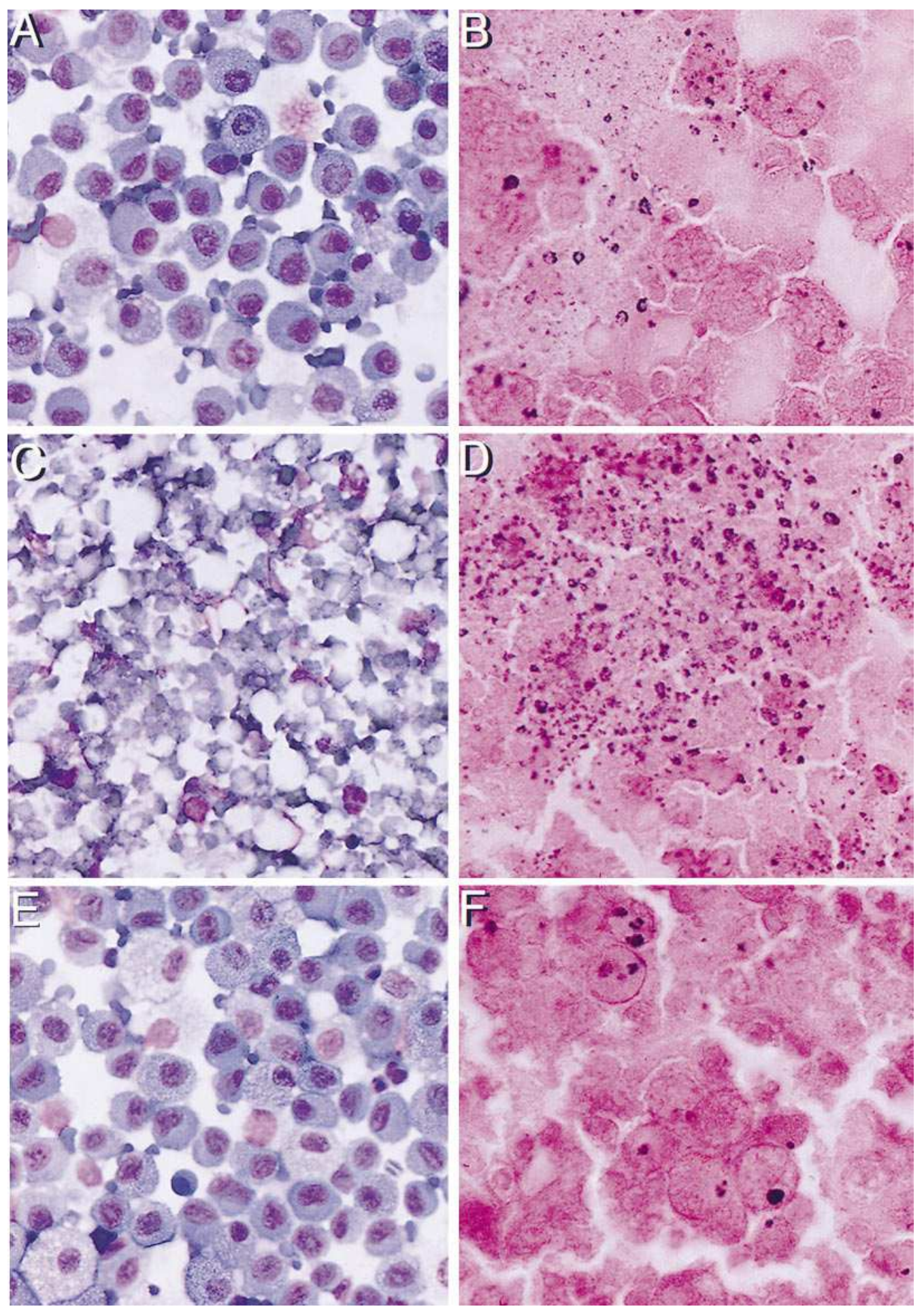

Figure 2. Liposomal dichloromethylene diphosphonate eliminates alveolar macrophages from the lung and impairs clearance of $P$. carinii.

$(A)$ Control rats were treated with intratracheal PBS, killed after $72 \mathrm{~h}$, and lower respiratory tract cellular populations assessed by bronchoalveolar lavage. Control rats treated with PBS exhibited typical lavage differential counts composed primarily of intact alveolar macrophages.

(Modified Wright Giemsa staining; $\times 100$.) $(B)$ Control rats that had received PBS

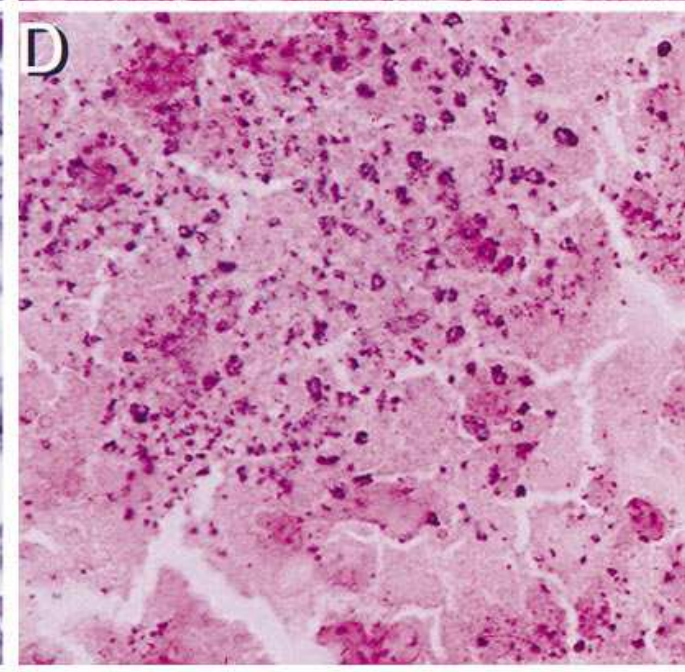
$72 \mathrm{~h}$ previously were inoculated with $P$. carinii $\left(1 \times 10^{7}\right)$. The next day, the rats were lavaged and $P$. carinii cysts burdens determined. Rats treated with PBS had effective elimination of $P$. carinii cysts. (Methenamine silver staining; $\times 160$. $)(C)$ Additional rats were treated with intratracheal L-Cl ${ }_{2} \mathrm{MDP}$, killed after $72 \mathrm{~h}$, and assessed by bronchoalveolar lavage. L- $\mathrm{Cl}_{2} \mathrm{MDP}$ treated rats had substantial reduction of alveolar macrophages. Numerous remnant macrophages consisting primarily of a condensed nuclear bodies with-

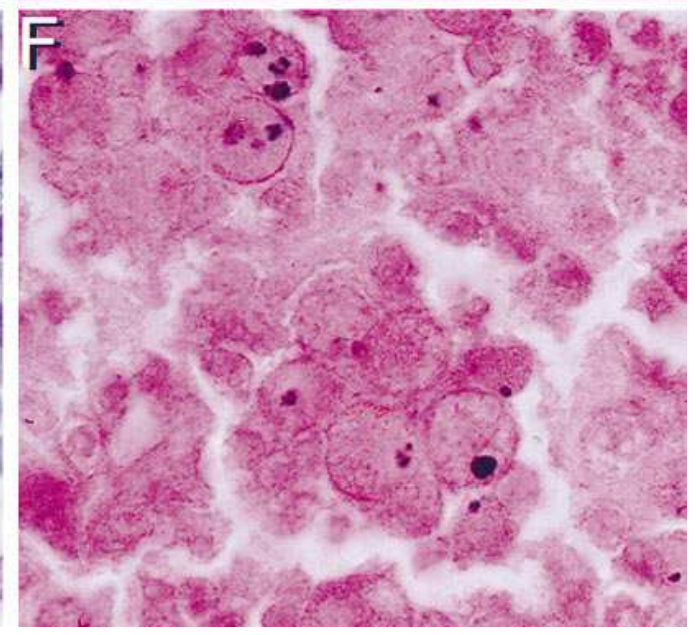
out definable cytoplasmic membranes were present. (Modified Wright Giemsa Staining; $\times 100$. $)(D)$ Rats depleted of macrophages with $\mathrm{L}-\mathrm{Cl}_{2} \mathrm{MDP} 72 \mathrm{~h}$ previously, were inoculated with $P$. cari$n i i$, and the cysts burdens determined the next day by bronchoalveolar lavage. Macrophage depleted rats had substantial impairment of $P$. carinii clearance.

(Methenamine silver staining; $\times 160$.) $(E)$ Rats treated with intratracheal L-PBS (liposome controls) exhibited lavage cellular differential counts predominantly containing normal macrophages. These cellular differentials of L-PBS treated rats were indistinguishable from rats receiving PBS alone. (Modified Wright Giemsa staining; $\times 100$.) $(F)$ Rats which had received L-PBS $72 \mathrm{~h}$ previously were inoculated with $P$. carinii, and the $P$. carinii cysts burdens determined the next day. Animals treated with L-PBS (liposome controls) also exhibited effective elimination of $P$. carinii from the lungs. (Methenamine silver staining; $\times 160$.)

brane, and characteristically consisted of only a condensed nuclear body. To further verify that the remnant macrophages and any residual macrophages were nonviable, in additional experiments, cells collected by bronchoalveolar lavage $72 \mathrm{~h}$ af- ter L-PAM administration were assessed for viability by the criterion that they contained a cytoplasm that could exclude trypan blue dye $(0.4 \%)$. In these experiments, remnant macrophages exhibited no definable cytoplasm capable of dye ex- 


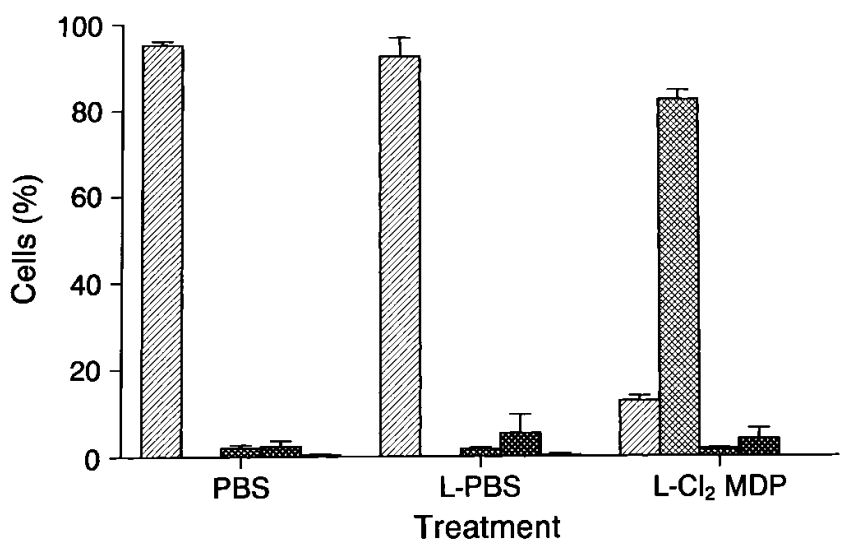

Figure 3. Liposomal dichloromethylene diphosphonate eliminates alveolar macrophages from the lung. Cellular differential counts obtained from whole-lung lavage of rats treated with either PBS, L-PBS, or L- $\mathrm{Cl}_{2} \mathrm{MDP}$. Rats treated with $\mathrm{L}-\mathrm{Cl}_{2} \mathrm{MDP}$ had substantial elimination of alveolar macrophages. In addition, macrophage elimination with $\mathrm{L}-\mathrm{Cl}_{2} \mathrm{MDP}$ did not result in significant recruitment of inflammatory cells including neutrophils. Shown is mean \pm SEM from three determinations. (Viable alveolar macrophages, ; remnant

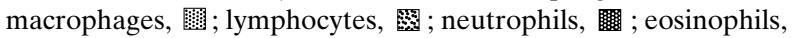

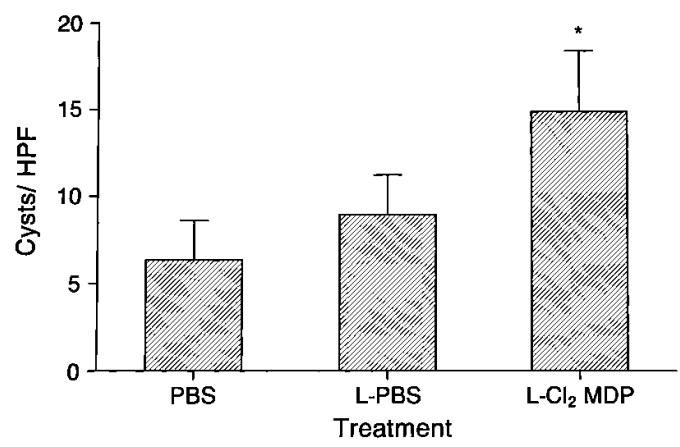

Figure 4. Rats depleted of macrophages have impaired clearance of $P$. carinii cysts. Rats received either PBS, L-PBS, or L-Cl $\mathrm{Cl}_{2} \mathrm{MDP}$. After $72 \mathrm{~h}$, the rats were again anesthetized and received transtracheal inoculation of $P$. carinii $\left(1 \times 10^{7}\right.$ organisms $) .24$ h later the rats were killed and whole-lung lavage performed. $P$. carinii cysts were counted in 40 contiguous high-powered fields on silver stained cytopreparation smears. Rats treated with $\mathrm{L}-\mathrm{Cl}_{2} \mathrm{MDP}$ had substantial impairment of $P$. carinii clearance as measured by higher levels of cysts counts in the lavage. ( ${ }^{*} P<0.05$ compared to PBS controls). Rats treated with L-PBS had only minimal impairment of $P$. carinii elimination, which was not significantly different from control rats receiving PBS alone. Shown is mean \pm SEM of three determinations.

den. To accomplish this, a mouse monoclonal antibody LM-1 was generated to purified gpA and characterized. LM-1, an IgG1k monoclonal antibody, reacted with purified gpA in immunoblot analysis (Fig. 5). LM-1 was subsequently used to develop a gpA ELISA to quantify $P$. carinii organism burden in whole lung BAL specimens. This ELISA detected the gpA content of 100 to 10,000 P. carinii organisms per sample of extract $(100 \mu \mathrm{l})$. Appropriate dilutions were performed to target the linear range of the assay in all subsequent studies.

The gpA ELISA was then used to further establish the role of macrophages in $P$. carinii clearance from the lower respiratory tract (Fig. 6). Rats were macrophage-depleted over $72 \mathrm{~h}$ by treatment with L-PAM and received transtracheal inoculation with purified $P$. carinii organisms. The next day $P$. carinii organism burden was assessed by BAL and gpA analysis. BAL

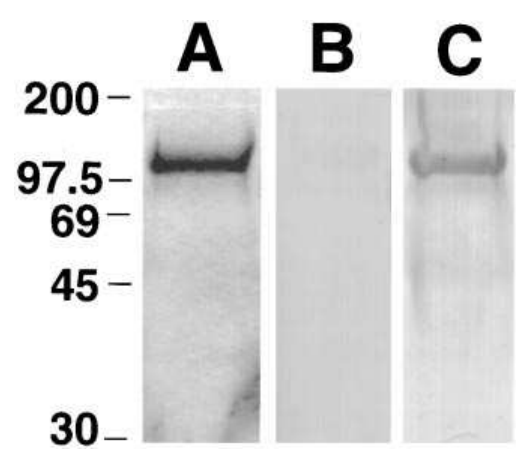

Figure 5. Generation of a mouse monoclonal antibody recognizing gpA, the major surface antigenic complex of $P$. carinii. Balb/c mice were immunized with purified gpA, the spleens fused with SP2/ OAg14 myeloma, and reactive cells cloned by limiting dilution to gen erate monoclonal antibody LM-1. (A) The

gpA antigenic complex was purified by continuous elution preparative gel electrophoresis. Shown is purified gpA complex (Coomassie Brilliant Blue staining) migrating at $\sim 120 \mathrm{kD}$. (B) Nonimmune mouse IgG failed to react with purified $P$. carinii gpA blotted onto nitrocellulose. $(C)$ Monoclonal antibody LM-1 reacted with the purified gpA protein complex. 


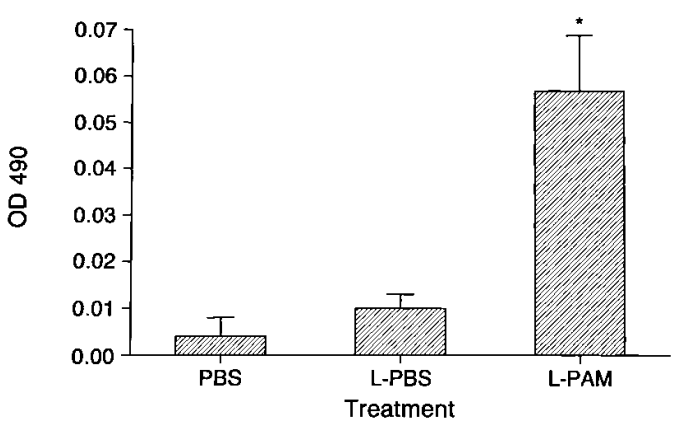

Figure 6. Determination of $P$. carinii burden in rats depleted of alveolar macrophages using a gpA ELISA. Rats received either intratracheal PBS, L-PBS, or L-PAM $(0.6 \mathrm{ml})$. After $72 \mathrm{~h}$ of macrophage depletion, the rats were transtracheally inoculated with $P$. carinii $(1 \times$ $10^{7}$ organisms). $24 \mathrm{~h}$ later, animals were killed, lavaged, and organism burdens determined using a gpA ELISA, as described in Methods. Rats treated with L-PAM had substantial impairment of $P$. carinii clearance as measured by higher levels of gpA in the lavage. $(* P<$ 0.05 compared to PBS control). Rats treated with L-PBS were not significantly different from controls receiving PBS alone. Shown is the mean \pm SEM of four determinations.

from macrophage-depleted rats exhibited 14.3 \pm 2.9 -fold greater gpA content compared to control rats who received PBS alone (PBS controls; $P=0.013$ ), and 8.1 \pm 1.7 -fold greater gpA content compared to rats who received L-PBS (liposomal controls; $P=0.008$ ). Rats who received L-PBS had minimally increased gpA content that was not significantly different than the gpA level found in BAL from PBS control rats $(P=0.24)$. These results further suggest that intact macrophage function mediates the degradation and clearance of $P$. carinii organisms from the lower respiratory tract.

\section{Discussion}

Recognition, uptake, and degradation of $P$. carinii by immune effector cells is essential for clearance of this organism from the lower respiratory tract. As a principal phagocyte occupying the airspaces, alveolar macrophages represent an integral component of host defense against this organism. The current study demonstrates that isolated alveolar macrophages possess the ability to not only bind and phagocytize $P$. carinii, but also to degrade and reduce the viability of the organism in culture. In addition, we demonstrate for the first time that macrophage function is necessary for clearance of $P$. carinii from the lungs of an intact mammalian host.

Before this study, methods to assess $P$. carinii degradation by alveolar macrophages are lacking in the literature. The current study provides a convenient method to monitor $P$. carinii degradation as ${ }^{35} \mathrm{~S}$-radiolabeled amino-acids are degraded and released into the culture media. Using this method, we observed significant degradation of $P$. carinii over $6 \mathrm{~h}$ of culture with macrophages. It is possible, however, that some ${ }^{35} \mathrm{~S}$-radiolabeled amino-acids released during degradation of $P$. carinii might be partially taken up and reincorporated into macrophage proteins. Such potential reutilization of ${ }^{35} \mathrm{~S}$-radiolabeled amino-acids by macrophages might underestimate $P$. carinii degradation, and the actual amount of $P$. carinii degraded by alveolar macrophages may possibly be even greater than re- ported. However, even if this was true, our conclusion that alveolar macrophages significantly degrade $P$. carinii in culture remains valid. The degradation of $P$. carinii and the impairment of its viability by alveolar macrophages in culture further supports a central role for these phagocytes in host defense against this organism.

Previous studies further indicate that cultured macrophages bind and phagocytize $P$. carinii organisms through specific receptor-ligand interactions. Ezekowitz et al. (17) demonstrated that macrophage mannose receptors mediate $P$. carinii uptake. Investigations from our laboratory indicate that macrophage mannose receptors recognize $P$. carinii by interacting with the mannose-rich gpA antigenic complex on the surface of the organism (20). Additional investigations indicate that the alveolar adhesive glycoproteins fibronectin, vitronectin, surfactant protein-D, and surfactant protein A bind to $P$. carinii and enhance interaction of the organism with macrophages (22, 23, 51-54). Ultrastructural studies have further suggested that $P$. carinii may be degraded within macrophages (13). However, these previous studies have not provided quantitative evidence of $P$. carinii degradation, nor have they investigated the effects of macrophage function on the organism within a living host.

Our current study evaluated the interaction of $P$. carinii with macrophages both in an isolated culture system, and further used the liposome suicide technique to analyze macrophage activity in the intact mammalian host. This latter method allows selective elimination of alveolar macrophages, and provides convincing evidence that macrophages degrade $P$. carinii and mediate organism clearance. This unique methodological approach should have wide applicability for studying macrophage function in a variety of lung diseases.

Our study provides a model of acute challenge and clearance of $P$. carinii from the lung by alveolar macrophages. Over the time course of these studies, no mortality was observed in macrophage-depleted animals challenged with $P$. carinii. Infection-related mortality was not observed in macrophagedepleted animals up to $10 \mathrm{~d}$ later. Assessing $P$. carinii-related mortality in macrophage-depleted animals over longer periods would be necessary to determine the role of alveolar macrophages in chronic models of $P$. carinii infection. Accordingly, additional experiments were performed in an attempt to chronically deplete rats by repeatedly injecting liposomal bisphosphonates at weekly intervals. However, on repeated injections, the lungs became infiltrated with neutrophils and monocytoid cells, and a chronic model of selective macrophage depletion without recruitment of additional inflammatory cells could not be established. Regardless, this study does provide firm evidence that alveolar macrophages can phagocytize and degrade $P$. carinii and substantially mediate the clearance of $P$. carinii from the lower respiratory tract after acute challenge with the organism.

Beyond the binding and phagocytosis of the organisms, alveolar macrophages are stimulated by $P$. carinii to release a number of inflammatory substances $(18,19,21,22)$. Interaction of surface-associated immunoglobulin on $P$. carinii with macrophage $\mathrm{Fc}$ receptors promotes the generation and release of eicosanoid metabolites (18). In contrast, fungal $\beta$-glucan in the $P$. carinii cell wall stimulates the macrophage release of reactive oxidant species and generation of the potent proinflammatory cytokine TNF $\alpha(19,21)$. TNF $\alpha$ expression is enhanced in macrophages recovered from patients with $P$. carinii pneu- 
monia, and is necessary for optimal elimination of $P$. carinii from the lungs of experimental animals $(55,56)$. Other studies further suggest that TNF $\alpha$ binds to the cell wall of $P$. carinii and may also exert a direct toxic effect upon the organism $(41,57)$.

Most clinical settings associated with $P$. carinii pneumonia are characterized by dysfunction of multiple components of immune surveillance including not only lymphocyte function, but also deficits in macrophages and other cells as well $(1,8$, 10). The function of alveolar macrophages is impaired in many conditions associated with the development of $P$. carinii pneumonia $(8,14,24)$. For instance, although HIV is well established to deplete CD4 lymphocytes, this agent also infects macrophages and monocytoid cells, resulting in impaired mannose receptor function and aberrant uptake of $P$. carinii (24). In addition, patients without AIDS who develop $P$. carinii pneumonia frequently do so as a complication of prolonged systemic corticosteroid therapy (8). Again, although corticosteroids have profound effects on lymphocytic immunity, these agents also substantially impair macrophage activation (10, 58-60). Functional impairment in the monocyte-macrophage system has also been documented in patients with advanced malignancies (61). Macrophage dysfunction may contribute in a synergistic fashion to other immunodepression such as CD4 lymphocytic immunity in promoting development of $P$. carinii pneumonia in various immunocompromised patient populations.

The quantification of organism burdens in patients and animal models of $P$. carinii pneumonia continues to be a challenging aspect of these types of investigations (1). Traditional methods based on counting cysts or trophozoites are time consuming, laborious, and may contain interobserver variability. Herein, we report a sensitive and specific ELISA-based assay to quantify $P$. carinii through measurement of gpA, its major surface antigen. $P$. carinii gpA is present on both cyst and trophozoite forms of the organism, and can serve as a marker for total numbers of organisms present within a given sample (20, 51). This method was applied to whole-lung BAL sampling, which gives a summary reflection of total organism burden in the $P$. carinii infected lung. This convenient method should prove useful for measuring $P$. carinii organism burden in other applications, such as in therapeutic drug trials, or in studies of $P$. carinii proliferation using in vitro culture systems.

In summary, we have demonstrated that alveolar macrophages bind, take up, and degrade $P$. carinii in tissue culture and also mediate clearance of the organisms from the lower respiratory tract of an intact host. The interactions of $P$. carinii organisms with alveolar macrophages represents a significant component of host recognition and defense against $P$. carinii infection.

\section{Acknowledgments}

We gratefully recognize the efforts of Dr. John A. McDonald and Ms. $\mathrm{Ka}$ Chen of the S.C. Johnson Research Center Immunology Core Facility, Mayo Clinic, Scottsdale, Arizona, who performed the immunization and fusion in the generation of monoclonal antibody LM-1. We also appreciate use of antibody 5E12 from Dr. Frank Gigliotti, University of Rochester. Further, we acknowledge the technical advice of Dr. Richard E. Pagano in the generation of liposomes. The authors appreciate the assistance of Drs. Yongmoon Han and Jim Cutler, of Montana State University, who provided initial samples of liposomal dichloromethylene diphosphonate, and Ms. Barbara Painter, of Miles Pharmaceuticals, for the gift of ciprofloxacin.

This work was supported by National Institutes of Health grants
R29AI-34336-03 and RO1HL-55934-01 to A.H. Limper. J.S. Hoyte was supported by funds from the National Medical Fellowship.

\section{References}

1. Limper, A.H., K.P. Offord, T.F. Smith, and W.J. Martin. 1989. Pneumocystis carinii pneumonia: differences in lung parasite number and inflammation in patients with and without AIDS. Am. Rev. Respir. Dis. 140:1204-1209.

2. Cohn, D.L., D.E. Stover, R.F. O'Brien, J.H. Shelhamer, T.A. Raffin, and P.C. Hopewell. 1988. Pulmonary complications of AIDS: advances in diagnosis and treatment. Am. Rev. Respir. Dis. 138:1051-1052.

3. Murray, J.G., and J. Mills. 1990. Pulmonary infectious complications of human immunodeficiency virus infection. Am. Rev. Respir. Dis. 141:1582-1598.

4. Sepkowitz, K.A., A.E. Brown, E.E. Telzak, S. Gottlieb, and D. Armstrong. 1992. Pneumocystis carinii pneumonia among patients without AIDS in a cancer hospital. JAMA (J. Am. Med. Assoc.). 267:832-837.

5. Peters, S.G., and U.B.S. Prakash. 1987. Pneumocystis carinii pneumonia. Am. J. Med. 82:73-78.

6. Centers for Disease Control. 1989. Guidelines for prophylaxis against Pneumocystis carinii pneumonia for persons with human immunodeficiency virus infection. MMWR (Morb. Mortal. Wkly. Rep.). 38:1-8.

7. Limper, A.H. 1991. Parasite adherence and host responses in the development of Pneumocystis carinii pneumonia. Semin. Respir. Infect. 6:19-26.

8. Yale, S.H., and A.H. Limper. 1996. Role of corticosteroids in development of Pneumocystis carinii pneumonia in patients without AIDS. Mayo Clin. Proc. 71:5-13.

9. Shellito, J., V.V. Suzara, W. Blumenfeld, J.M. Beck, H.J. Steger, and T.H. Ermak. 1990. A new model of Pneumocystis carinii infection in mice selectively depleted of helper T lymphocytes. J. Clin. Invest. 85:1686-1693.

10. Masur, H., H.C. Lane, J.A. Kovacs, C.J. Allegra, and J.C. Edman. 1989. NIH conference. Pneumocystis pneumonia: from bench to clinic. Ann. Intern Med. 111:813-826.

11. Beck, J.M., M.L. Warnock, J.L. Curtis, M.J. Sniezek, S.M. Arraj-Peffer, H.B. Kaltreider, and J.E. Shellito. 1991. Inflammatory responses to Pneumocystis carinii in mice selectively depleted of helper T lymphocytes. Am. J. Respir. Cell Mol. Biol. 5:186-197.

12. Von Behren, L.A., and E.L. Pesanti. 1978. Uptake and degradation of Pneumocystis carinii by macrophages in vitro. Rev. Respir. Dis. 118:1051-1059.

13. Masur, H., and T.C. Jones. 1978. The interaction in vitro of Pneumocystis carinii with macrophages and L-cells. J. Exp. Med. 147:157-170.

14. Koziel, H., D. O'Riordan, A. Warner, and R.M. Rose. 1994. Alveolar macrophage interaction with Pneumocystis carinii. Immunol. Ser. 60:417-436.

15. Laursen, A.L., N. Obel, J. Rungby, and P.L. Anderson. 1993. Phagocytosis and stimulation of the respiratory burst in neutrophils by Pneumocystis carinii. J. Infect. Dis. 168:1466-1471.

16. Mason, G.R., C.H. Hashimoto, P.S. Dickman, L.F. Foutty, and C.J. Cobb. 1989. Prognostic implications of bronchoalveolar lavage neutrophilia in patients with Pneumocystis carinii pneumonia and AIDS. Am. Rev. Respir. Dis. 139:1336-1342.

17. Ezekowitz, R.A.B., D.J. Williams, H. Kozial, M.Y.K. Armstrong, A. Warner, F.F. Richards, and R.M. Rose. 1991. Uptake of Pneumocystis carinii mediated by the macrophage mannose receptor. Nature (Lond.). 351:155-158.

18. Castro, M., T.I. Morgenthaler, O.A. Hoffman, M.S. Rohrbach, and A.H. Limper. 1993. Pneumocystis carinii induces the release of arachidonic acid and its metabolites from alveolar macrophages. Am. J. Respir. Cell. Mol. Biol. 9:73-81.

19. Hidalgo, H.A., R.J. Helmke, V.F. German, and J.A. Mangos. 1992. Pneumocystis carinii induces an oxidative burst in alveolar macrophages. Infect. Immun. 60:1-7.

20. O'Riordan, D.M., J.E. Standing, and A.H. Limper. 1995. Pneumocystis carinii glycoprotein A binds macrophage mannose receptors. Infect. Immun. 63: 779-784.

21. Hoffman, O.A., J.E. Standing, and A.H. Limper. 1993. Pneumocystis carinii stimulates tumor necrosis factor- $\alpha$ release from alveolar macrophages through a $\beta$-glucan-mediated mechanism. J. Immunol. 150:3932-3940.

22. Neese, L.W., J.E. Standing, E.J. Olson, M. Castro, and A.H. Limper. 1994. Vitronectin, fibronectin, and gp120 antibody enhance macrophage release of TNF-alpha in response to Pneumocystis carinii. J. Immunol. 152:4549-4556.

23. Pottratz, S.T., and W.J. Martin. 1990. Mechanism of Pneumocystis carinii attachment to cultured rat alveolar macrophages. J. Clin. Invest. 86:16781683.

24. Koziel, H., B.A. Kruskal, R.A. Ezekowitz, and R.M. Rose. 1993. HIV impairs alveolar macrophage mannose receptor function against Pneumocystis carinii. Chest. 103(Suppl):111S-112S.

25. Kovacs, J.A., F. Powell, J.C. Edman, B. Lundgren, A. Martinez, B. Drew, and C.W. Angus. 1993. Multiple genes encode the major surface glycoprotein of Pneumocystis carinii. J. Biol. Chem. 268:6034-6040.

26. Gigliotti, F. 1992. Host species-specific variation of a mannosylated surface glycoprotein of Pneumocystis carinii. J. Infect. Dis. 165:329-336.

27. Linke, M.J., M.T. Cushion, and P.D. Walzer. 1989. Properties of the major antigens of rat and human Pneumocystis carinii. Infect. Immun. 57:1547- 
1555 .

28. Radding, J.A., M.Y.K. Armstrong, E. Ullu, and F.F. Richards. 1989. Identification and isolation of a major cell surface glycoprotein of Pneumocystis carinii. Infect. Immun. 57:2149-2157.

29. Cushion, M.T., J.A. DeStefano, P.D. Walzer. 1988. Pneumocystis carinii: surface reactive carbohydrates detected by lectin probes. Exp. Parasitol. 67: $137-147$.

30. Haidaris, P.J., T.W. Wright, F. Gigliotti, and C.G. Haidaris. 1992. Expression and characterization of a cDNA clone encoding an immunodominant surface glycoprotein of Pneumocystis carinii. J. Infect. Dis. 166:1113-1123.

31. Stringer, S.L., T. Garbe, S.M. Suskin, and J.R. Stringer. 1993. Genes encoding antigenic surface glycoproteins in Pneumocystis carinii in humans. J. Eukaryot. Microbiol. 40:821-826.

32. Lundgren, B., G.Y. Lipchik, and J.A. Kovacs. 1991. Purification and characterization of a major human Pneumocystis carinii surface antigen. J. Clin. Invest. 87:163-170.

33. Kolls, J.K., J.M. Beck, S. Nelson, W.A. Summer, and J. Shellito. 1993. Alveolar macrophage release of tumor necrosis factor-alpha during murine Pneumocystis carinii pneumonia. Am. J. Resp. Cell Mol. Biol. 8:370-376.

34. Theus, S.A., M.J. Linke, R.P. Andrews, and P.D. Walzer. 1993. Proliferative and cytokine responses to a major surface glycoprotein of Pneumocystis carinii. Infect. Immun. 61:4703-4709.

35. Gigliotti, F., D.C. Stokes, A.B. Cheatham, D.S. Davis, and W.T.

Hughes. 1986. Development of murine monoclonal antibodies to Pneumocystis carinii. J. Inf. Dis. 154:315-322.

36. Qian, Q., M.A. Jutila, N. Van Rooijen, and J.E. Cutler. Elimination of mouse splenic macrophages correlates with increased susceptibility to experimental disseminated candidiasis. J. Immunol. 152:5000-5008.

37. Limper, A.H., and W.J. Martin. 1990. Pneumocystis carinii: inhibition of lung cell growth mediated by parasite attachment. J. Clin. Invest. 85:391-396.

38. Bartlett, M.S., J.A. Fishman, S.A. Queener, M.M. Durkin, M.A. Jay, and J.W. Smith. 1988. New rat model of Pneumocystis carinii pneumonia. J. Clin. Microbiol. 26:1100-1102.

39. Limper, A.H., S.T. Pottratz, and W.J. Martin. 1991. Modulation of Pneumocystis carinii adherence to cultured lung cells by a mannose-dependent mechanism. J. Lab. Clin. Med. 118:492-499.

40. Limper, A.H., J.E. Standing, O.A. Hoffman, M. Castro, and L.W. Neese. 1993. Vitronectin binds to Pneumocystis carinii and mediates organism attachment to cultured lung epithelial cells. Infect. Immun. 61:4302-4309.

41. Pesanti, E.L., T. Tomicic, and S.T. Donta. 1991. Binding of ${ }^{125}$ I-labeled tumor necrosis factor to Pneumocystis carinii and an insoluble cell wall fraction. J. Protozool. 38:28S-29S

42. Bartlett, M.S. P.A. Vervanac, and J.W. Smith 1979. Cultivation of Pneumocystis carinii with WI-38 cells. J. Clin. Microbiol. 10:796-799.

43. McDonald, J.A., B.J. Quade, T.J. Broekelmann, R. LaChance, K. Forsman, E. Hasegawa, and S. Akiyama. 1987. Fibronectin's cell-adhesive domain and an amino-terminal matrix assembly domain participate in its assembly into fibroblast pericellular matrix. J. Biol. Chem. 262:2957-2967.

44. McDonald, J.A., T.J. Broekelmann, M.L. Matheke, E. Crouch, M. Koo, and C. Kuhn III. 1986. A monoclonal antibody to the carboxyterminal domain of procollagen type I visualizes collagen-synthesizing fibroblasts. Detection of an altered fibroblast phenotype in lungs of patients with pulmonary fibrosis. $J$.
Clin. Invest. 78:1237-1244

45. Armstrong, M.Y.K., H. Koziel, R.M. Rose, C. Arena, and F.F. Richards 1991. Indicators of Pneumocystis carinii viability in short term culture. J. Protozool. 38:88S-89S.

46. Berg, J.T., S.T. Lee, T. Thepen, C.Y. Lee, and M.F. Tsan. 1993. Depletion of alveolar macrophages by liposome-encapsulated dichloromethylene diphosphonate. J. Appl. Physiol. 74:2812-2819.

47. Van Rooijen, N. 1989. The liposome-mediated macrophage "suicide" technique. J. Immunol. Method. 124:1-6.

48. Monkkonen, J., N. van Rooijen, and P. Ylitalo. 1991. Effects of clodronate and pamidronate on splenic and hepatic phagocytic cells of mice. Pharm. Toxicol. 68:284-286.

49. Monkkonen, J., H.M. Koponen, and P. Ylitalo. 1990. Comparison of the distribution of three bisphosphonates in mice. Pharm. Toxicol. 66:294-298.

50. Limper, A.H., U. Specks, W.M. Brutinel, W.J. Martin II, and M.S Rohrbach. 1993. Interlobular variation in the recovery of bronchoalveolar lavage fluid, cell populations, and angiotensin-converting enzyme in normal volunteers. J. Lab. Clin. Med. 121:785-791.

51. O'Riordan, D.M., J.E. Standing, K.Y. Kwon, D. Chang, E.C. Crouch, and A.H. Limper. 1995. Surfactant protein D interacts with Pneumocystis carinii and mediates organism adherence to alveolar macrophages. J. Clin. Invest. 95:2699-2710.

52. Phelps, D.S., and R.M. Rose. 1991. Increased recovery of surfactan protein A in AIDS-related pneumonia. Am. Rev. Respir. Dis. 143:1072-1075.

53. Zimmerman, P.E., D.R. Voelker, F.X. McCormack, J.R. Paulsrud, and W.J. Martin. 1992. 120-kD surface glycoprotein of Pneumocystis carinii is a ligand for surfactant protein A. J. Clin. Invest. 89:143-149.

54. Williams, M.D., J.R. Wright, K.L. March, and W.J. Martin II. 1996. Human surfactant protein A enhances attachment of Pneumocystis carinii to rat alveolar macrophages. Am. J. Respir. Cell Mol. Biol. 14:232-238.

55. Krishnan, V.L., A. Meager, D.M. Mitchell, and A.J. Pinching. 1990. Alveolar macrophages in AIDS patients: increased spontaneous tumour necrosis factor-alpha production in Pneumocystis carinii pneumonia. Clin. Exp. Immunol. 80:156-160.

56. Chen, W., E.A. Havell, and A.G. Harmsen. 1992. Importance of tumor necrosis factor-alpha and gamma interferon in host resistance against Pneumocystis carinii infection. Infect. Immun. 60:1279-1284.

57. Pesanti, E.L. 1991. Interaction of cytokines and alveolar cells with Pneumocystis carinii in vitro. J. Infect. Dis. 163:611-616.

58. Walzer, P.D., M. LaBine, T.J. Redington, and M.T. Cushion. 1984. Lymphocyte changes during chronic administration and withdrawal of corticoste roids: relevance to Pneumocystis carinii pneumonia. J. Immunol. 133:25022508 .

59. Siminski, J., P. Kidd, G.D. Phillips, C. Collins, and G. Raghu. 1991. Reversed helper/suppressor T-lymphocyte ratio in bronchoalveolar lavage from patients with breast cancer and Pneumocystis carinii pneumonia. Am. Rev. Respir. Dis. 143:437-440.

60. Huang, Z.B., and E. Eden. 1993. Effect of corticosteroids on IL1 beta and TNF alpha release by alveolar macrophages from patients with AIDS and Pneumocystis carinii pneumonia. Chest. 104:751-755.

61. Garraud, O., A. Faucher, and E. Legrand. 1988. Impairment of monocyte functions in advanced head and neck cancer. Immunol. Lett. 18:213-218. 